INFORMAL REPORT

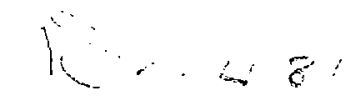

$\mu$-Capture and the $n-n$ Scattering Length

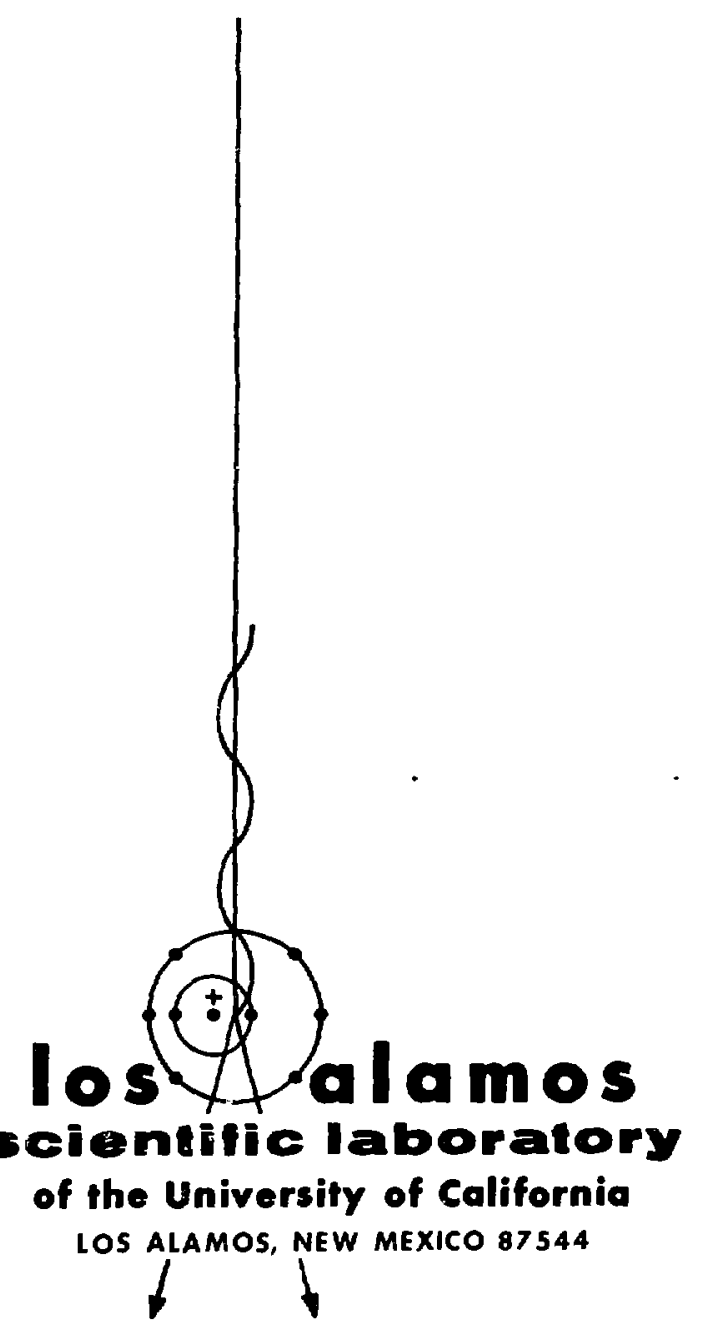


This repon wes prepered as an secount of work sponsored by the United Stutes Government. Neither the United States nor the United Stutes Atomic Energy Commiaion, nor any of their employem, nor any of their contrectors, ubeontractors, or their employees, makes any warranty. express of implied, or anumes eny lepal liabllity or responsibility for the eccursecy. com. pleteness or usefulnes of eny information, apperatus, product or process dis. closed, or represents that its use woukd not infringe peivately owned rights.

In the interest of prompt distribution. this LAME report was not edited by the Technical Information stalf.

Printed in the United States of America. Available from

National Technical Intormation Service

U. S. Departinent of Commerce 5285 Port Povel Rowy

Springlield. Virginia 22151

Price: Printed Copy S4.00; Mieroliche \$1.45 
LA-5424-MS

Informal Report

UC.34c

ISSUED: Noveinber 1973

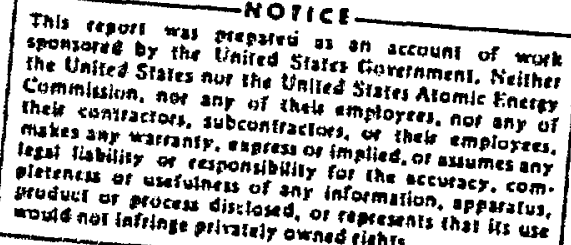

\section{$\mu$-Capture and the n-n Scattering Length}

by

B. F. Gibson

G. J. Stephenson, Jr."

* John Simon Guggenheim Fclluw. Permanent address: Department of Physics. University of Maryland, College Fark. MO 20742.

This work partially supported by the US Atomic Energy Commission's Division of Physical Research. 
w-Capture ard the $n-n$ Scatter1ng Lenth

by

B. F. Gibson and G. J. Stephenson, Jr.

\section{ABSTRAT}

The capture reaction $u^{-}+d \rightarrow n+n+v$ is exnmined with the objective of obtsining a precice pessuresent of the $n-n$ scattering length. Differential time-of-IIIght neutron spectra for the twaneutron colneldence experiment are calculated to 1llustrate the oensitivity of the reaction to $\mathrm{Ann}$. The doainant theoretieai uncertainsy I1es in the uiknown short-range character of the $n-n$ gcattering rave runetion os it does for cospetsing reactions such as radiative $\pi$-capture.

\section{DITHODUCTION}

It has been recognized for some the that the nucleon-mucleon (N-N) interaction is not charge Independent: i.e., the n-p force is not the sabe as elther the $n-n$ force or the nuelear part of tho $\mathrm{p}-\mathrm{p}$ force. However, the question of charge eymetry-cquality of the $n=n$ force and the nuclear part of the p-p force-nas not been settled. It is a question of rundamental interest to phyoles. Because the acattering lengtho are lorge, seall differences in the $X-n$ interactions wil irduce sizeable differences in the respective scaterine lengths. Hence a precise seasuresent of the n-n and p-p scattering lengths is a good wethod of exanining the question of charge asymetry. The p-p ncattering length is well deterwined, but the uncertainty in the $n-n$ scattering lensth 15 of the order of $\pm I$ (see Appendix $A$ ). Therefore, we wish to examine here the possibility of obtaining o precise value of the $n-n$ scattering length an from the $\mu$-capture reaction.

Because the reak interaction is thought to be reasonably rell understood, the capture reaction

$$
u^{-}+d-n+n+v
$$

chould be wuch wre easily interpreted than either of the reactions:

$$
\begin{aligned}
& n^{-}+a-n+n+\gamma \\
& n+a+n+n+p .
\end{aligned}
$$

If Indeed the conscrved-vector-curient theory (CYC) Is val1d, then half of the ontrix elesents involved in the general veak internction, those of the vector curreat, are cxactly the sace as thosu which are veasted in electron scattering. I lke bine electromatnctic force, the veah fozce to doainated by a sifalc interaction, so that tion wurtiple acntterinc effects are neelleible. '17us, the $\mu-$ capture process, betng one in which there ore only two strongly interating particles in either tite

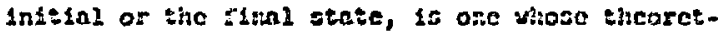
scal interpretat ios should be well fousted. Screrol calculations or the is-capture rate if desteritu have bees reported. ${ }^{1-6}$ Resulto obtnincd cerpare covorably usth the expericental capture rate $i$ of approxientely $320 \mathrm{sec}^{-1}$. The reacomble ofreescrt of the theoret teal and expeysretital rntes ouds to our sonfluence that the calculated ncutron apesern Wh1 provide realletic estiontco as a buide so the experiachtaist.

llowever, it should be noted here that the largect uncertulnty in the acterfinntion of $a_{\mathrm{ma}}$ rrom the rodlative $a$-capture renetson cones not 
from the uncertainty in the Initial state but from the unkuom short-range character of the flnalstate $n-n$ wave function. The $\mu$-capture reaction w11l suffer the same source of uncertainty in the final state, and therefore, it may not offer as much of a theoretical edvantage as the above arguwents would imply. (See Appendix B.)

\section{MERXREIICAT STMAAKY}

If wo denote the veais interaction Familtonian by $A$, ara use first-order perturbation theory, then we can weite the probab111ty for $\mu$-capture as

$$
d w=(2 \pi)^{-5} \delta_{4}\left(p_{1}-p_{f}\right) \Sigma\left|\left(f\left|F_{W}\right| 1\right\rangle\right|^{2} d^{3} p_{1} d^{3} p_{e} a^{3} v,
$$

where $\vec{p}_{1}, \ddot{p}_{2}$, and $\vec{v}$ are the three wonta of the tro neutrons and the neutrinc, respectively, $\left(\vec{P}_{f}=\vec{P}_{1}+\vec{P}_{2}+\vec{v}\right)$, and $P_{1}$ and $P_{f}$ are the initial and final four womenta. The weal intexaction

Familtonian 18 asemed to be of the current-current Interection fosm

$$
H_{W}=-\frac{G}{2} \int \partial_{\lambda}^{(+)}(x) \partial_{\lambda}^{(-)}(x) \mathrm{d}^{3} x
$$

where the current 16 the sum of hadronlc and leptoric parts:

$$
\partial_{\lambda}^{(-)}=\partial_{\lambda}^{(-)} \text {(hadron) }+j_{\lambda}^{(-)} \text {(1epton) . }
$$

The coupling constant $G$ is given by $O=10^{-5} / \mathrm{m}^{2}$, where of 10 the proton mas. The general $V$ - A Iepton current can be expressed as

$$
j_{\lambda}^{(-)}=1\left[\tau_{e-1} \gamma_{\lambda}\left(1+\gamma_{5}\right) t_{v_{e}}+\tau_{\mu}-\gamma_{\lambda}\left(1+\gamma_{5}\right) v_{v_{\mu}}\right]
$$

wher F and t are the relativiotic quantum Ilelds describing the leptons, We have written here the rull lepton current in orier to ouphsize the aimilarity between the election and the wuon weak currents which lead to i-decay, e-copture, H-cspture, etc.

By restricting our intereat to just the deuteron H-capture reaction, the weak Hamiltonian may be reduced to the following nonrelotivastic effective interaction Hanlitonien: $8-10$

$$
\begin{aligned}
\mathrm{Z}_{W}=\frac{G}{2} \sum_{1} \tau_{1}\left[G_{V}\right. & +\left[G_{P} \vec{\sigma}_{1} \cdot \hat{v}+\left(F_{A} / m\right) \vec{p}_{1} \cdot \vec{\sigma}_{1}\right] \sigma_{\ell} \cdot \vec{v} \\
& \left.+\left[G_{A} \vec{\sigma}_{1}+\left(F_{V} / m\right) \vec{p}_{1}\right] \cdot \vec{\sigma}_{\ell}\right]\left(1-\vec{\sigma}_{\rho} \cdot \hat{v}\right)
\end{aligned}
$$

where the subscript 1 denotes mucleon operators and the subscript $\ell$ denotes lepton operators. The normblized coupling constants are:

$$
\begin{aligned}
& G_{V}=\left(1+\frac{v}{2 m}\right) F_{V} \\
& G_{A}=F_{A}-\left(1+\mu_{P}-\mu_{n}\right) \frac{v}{2 m} F_{V} \\
& G_{P}=\left(F_{P}-F_{A}-\left(1+\mu_{P}-\mu_{n}\right) F_{V}\right) \frac{v}{2 m} \\
& F_{V}=1 . \\
& F_{A}=-1,24 F_{V} \\
& F_{P}=7 F_{A} .
\end{aligned}
$$

The subacripts $V, A$, and $P$ denote the rector, axiel rector, and Induced pseudoscalar coupling, $\mu_{p}$ and $\mu_{n}$ are the anowalous moments of the proton and the neutron, and $\tau_{f}^{-}$is the nucleon 180eptn lowering operator. (The pseudoscalar coupling $F_{p}$ is not well determined, but the effect of 1ts uncertainty on the neutron apectra is rather sanall.)

Oaing this form of the errective weak Hamiltonian, we can write the appropriate spin-averaged square of the mertx element corrosponding to the $\mu-d$ atom as

$$
\begin{aligned}
& \mid\langle e| \text { H }\left.|1\rangle\right|^{2} \\
& \sim_{i} \sum_{m_{f}}\left\{G_{V}^{2}\left|\left\langle m_{f}\left|\Sigma \tau_{i}^{*} e^{1 \vec{v} \cdot \vec{r} / \partial_{\varphi_{\mu}}}(r)\right| m_{i}\right)\right|^{2}\right. \\
& +G_{A}^{2}\left|\left(m_{l}\left|\sum_{1}^{\Sigma} \tau_{i}-\overrightarrow{\sigma_{1}} e^{1 \vec{v} \cdot \vec{z} / 2} \varphi_{\mu}(r)\right| m_{1}\right\rangle\right|^{2} \\
& +\left(\sigma_{p}^{2}-2 \sigma_{p} q_{A}\right)\left|\left(m_{p}\left|\sum \tau_{1}^{-} \vec{\sigma}_{1} \cdot \hat{v} e^{1 \vec{v} \cdot \vec{r} / 2} q_{\mu}(r)\right| \sigma_{1}\right\rangle\right|^{2} \\
& \text { - } G_{v} q_{v} \text { 2Re}\left[\left(m_{p}\left|\Sigma \tau_{1}^{-} e^{1 \vec{v} \cdot \vec{r} / 2} \varphi_{\mu}(r)\right| m_{1}\right\rangle\right. \\
& \text { * } \left.\left(m_{f}\left|\Sigma \tau_{1}^{*} e^{1 \vec{v} \cdot \vec{r} / \partial_{\varphi_{\mu}}(r)} \partial \cdot \vec{p}_{i} / m\right| m_{1}\right)\right] \\
& -\left\langle G_{A} B_{A}-G_{P} B_{A}\right) \operatorname{2Re}\left[\left\langle\nabla_{P}\right| \Sigma \tau_{1}^{-} e^{1 v \cdot r / \partial_{\varphi_{\mu}}(r)\left|w_{1}\right\rangle}\right. \\
& \left.\left.{ }^{u}\left\langle\omega_{1}\left|\sum_{1} \tau_{1}^{-} e^{1 \vec{v} \cdot \vec{r} / 2} \varphi_{\mu}(r) \vec{\sigma}_{1} \cdot \vec{p}_{1} \cdot m\right| m_{1}\right\rangle\right]\right\}
\end{aligned}
$$


where the latter two terms describe the so-called relativistic corrections to order $p_{1} / m$. The intrix element is dominated by the secont term, which contains the triplet-singlet transition. This is most easily seen in the S-state approximation for the deuteron, where the result (neglecting the relatiristic corrections) reduces to

$$
\begin{aligned}
\left|\left\langle f\left|H_{w}\right| i\right\rangle\right|^{2} & \sim G_{V}^{2} I_{t}^{2}+G_{A}^{2}\left(2 I_{t}^{2}+I_{s}^{2}\right) \\
& +\left(G_{P}^{2}-2 G_{A} G_{P}\right) \frac{1}{3}\left(2 I_{t}^{2}+I_{g}^{2}\right) .
\end{aligned}
$$

The $I_{s}$ and $I_{t}$ denote integrals over the triplet and singlect final spin states of the two neutrons. It is $I_{s}$ that contains the strong final-state s-wave interaction. Because $G_{p}$ is proportional to $v / m$ it becomes a small correction to the $G_{A}^{2}$ contribution.

Becouse the contribution of the relativistic corrections to the totai capture rates is small, we shall neglect their contributions to the spectra in the curves presented below. The approximution is unimportant for our demonstration pirposes, although they would be included in an actual analysis. It should also be pointed out that although $G_{A}$ and $G_{P}$ contain a momentum dependence through $v$, the momentus $v$ is effectively a constant $\left(\nu \approx \nu_{\max }\right)$ over the kinematic region in which we are interested.

The general form of the radial integrals that arise can be represented schematicaly as

$$
I=\int_{\varphi_{\mu}}(r) \varphi_{d}(r) e^{i \vec{v} t ; / 2} \varphi_{n n}(p, r) d^{3} r
$$

where $\varphi_{d}$ is the deuteron bound state, ${ }^{n}$ is the two-neutron scattoring state, $\exp (i \vec{v} \cdot \vec{r} / 2)$ represents the neutrino, and $\varphi_{\mu}$ is the atonic muon wave function. We have used a Reid soft-core deuteron bound state including the D-state. For the $n-n$ scattering stace we have used a PRS wave function ${ }^{11}$ in the singlet s-state, which perwits us to vary the short-range charecter of the final state while holding the scattering length and efo. rective runge fixed. We uish to empherize that the use of zero-range deuteron wave Dunrtions and asymptotic surms of the final stato such as

$$
\varphi_{n n}(p, r) \sim\left[j_{0}(p r) \cos \delta-n_{0}(p r) \sin \delta\right]
$$

san lead to error in exirecting a from an experimental spectrum. We have assumed throughout that the $\mu$ is captured from an $s$ atomic orbit. This should be a bood approximation since the $\mathrm{Hd}$ atcon is a one-electron atom, and stark-effect transiticns as jure that the predominant capture comes frool s-orbitals. 12

\section{NUMERICAL RESUITS}

Beceuse measurement of an absolute cross section is difficult, we onit absolute normalizations from our results. Instexd we study what can be learned from a measuremert of the neitron spectra in a differential time-of flight experiment. (It is clear that one cannot make a normal two-neutron coincidence measurement, as in the case of radiative $\pi$-capture, since one cannot detect the $v$ as $e$ start pulee.)

The spectra of interest are of the form

$$
\begin{aligned}
\frac{d \sigma}{d \Omega_{1} d \Omega_{2} d(\Delta t)} & =\frac{d \sigma}{d \Omega_{1} d \Omega_{2} d E_{1}} \frac{d E_{1}}{d(\Delta t)} \\
& =\frac{\left|\left\langle H_{W}\right\rangle\right|^{2} p_{1} p_{2}}{f^{\prime}} \frac{d E_{1}}{d(\Delta t)},
\end{aligned}
$$

where $p_{1}, p_{2}$, are the two neutron momenta, $f^{\prime}$ is the recoil factor, and $d E_{1} / d(\Delta t)$ is the transformation Jacobian from $E_{1}$ to the differential time of elight $\Delta t$. The product of $a E_{1} / d(\Delta t)$ and $I / f^{\prime}$ is given by

$$
\begin{aligned}
\frac{1}{r} \frac{d F_{1}}{d(\Delta t)} \sim \mid & p_{1}^{-3}\left[1+\frac{q}{v}\left(1+\frac{p_{1}}{p_{2}} \cos \theta\right)\right] \\
& +\left.p_{2}^{-3}\left[1+\frac{p}{v}\left(1+\frac{p_{2}}{p_{1}} \cos \theta\right)\right]\right|^{-1}
\end{aligned}
$$

and is clearly symetric in the two neutron. momenta Because we wish to make the relative momentun $\vec{p}=\left(\vec{p}_{2}-\vec{p}_{1}\right) / 2$ small to incure that the $n-n$ scattering length dominoted the finol-state interaction, the opening angle ${ }_{1} / 2$ between tine two neutrons should be small. We illustrate the expected spectra normalized to unit area for $A_{12}=5^{\circ}, 10^{\circ}$, and $20^{\circ}$ in Figs. 1-3. It is apparent 


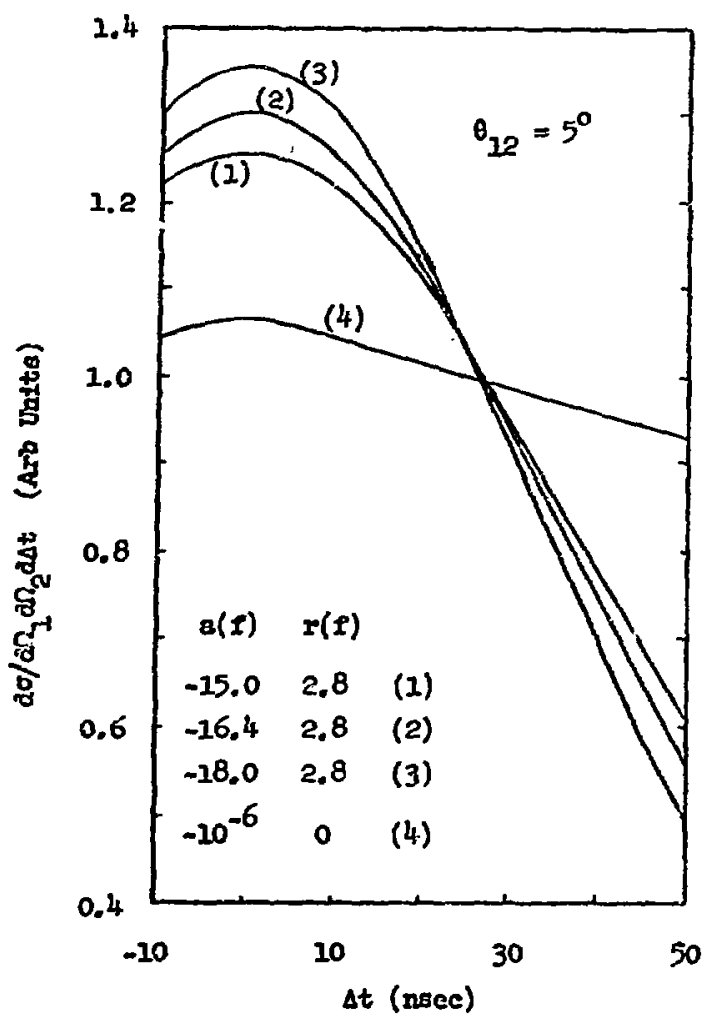

Fig. 1. Tro-neutron afferential time-of-flight spectra normallzed to unit area over the symetric interval $-50<\Delta t<+50$ plotted for $\theta_{12}=5^{\circ}$ and various values of a . Curve (4) corresponds to phase space $^{\mathrm{n}}$. Flight path equal $3 \mathrm{~m}$.

that differentiating between $a_{n n}=-16.4 f$ and either $-18 f$ or $-15 f$ is guite feasible. However, determining $a_{n n}$ to $\pm 0.3 f$ will obviously require large statistics and a good statigtical onatysts.

For comarison with the sensitivity of the apectrum to an we have shown in Fig. 4 the sensicfurcy of the spectrum to variation in the unknown effective range $r_{n n}$. Only results for an opening angle of $20^{\circ}$ are presented, since differences at $5^{\circ}$ and $10^{\circ}$ could not be been on the scale plotted. Although the effects due to yarlation in $r_{n n}$ are emall compared to those due to a comparable percentage variation in $a_{n n}$, the fact that $r_{n n}$ is not known becomes a non negligible factor for $\theta_{12}>15^{\circ}$, such that $p$ becomes non-negl1Bible.

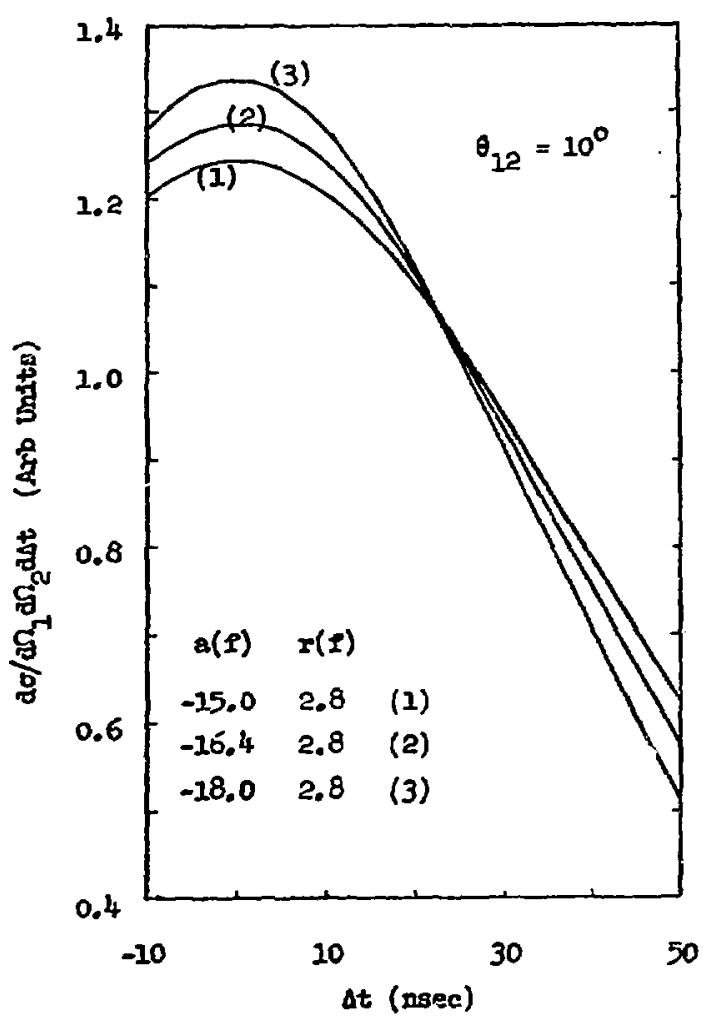

Fig. 2. Tro-neutron differential time-of-flight spectra normalized to unit area over the symetric interral $-50<\Delta t<+50$ plotted for $\theta_{12}=10^{\circ}$ and various values of $a_{n n}$. Fitight path equal $3 \mathrm{~m}$.

Clearly the correct choice of experimental variables is important. The opening angle determines the minimum value of the relative momentum, $p_{\min }$. The time interval $\Delta t$ determines the range of allowed $p$ and therefore $p_{\max }$. One would like $P_{\min }$ to be less than $1 / \mathrm{a}_{\mathrm{nn}}$ or gbout $12 \mathrm{MeV} / \mathrm{c}$. Thus the opening angles of $5^{\circ}-20^{\circ}$ would be suitable. However, as noted above, for $\theta_{12}>15^{\circ}$ the effective range can be a problem. Also, as the opening angle is decreased, the range in $p$, for a given interval in $\Delta t$, Increases: $\Delta t= \pm 50 \mathrm{nsec}$ implies $4.4 \mathrm{MeV} / \mathrm{c} \leqslant \mathrm{p}<7.8 \mathrm{MeV} / \mathrm{c}$ at $10^{\circ}$ compared with $2.2 \mathrm{MeV} / \mathrm{c}<\mathrm{p}<6.8 \mathrm{MeV} / \mathrm{c}$ at $5^{\circ}$. However, one would like to have $p_{\max }$ as large as posstble in order to make the spectrum sensitive to small variation in $a_{n n}$. Thus a compromise between the largest reasonable range in $p$ and the largest reasonable value of $p_{\max }$ would seem to inply an 


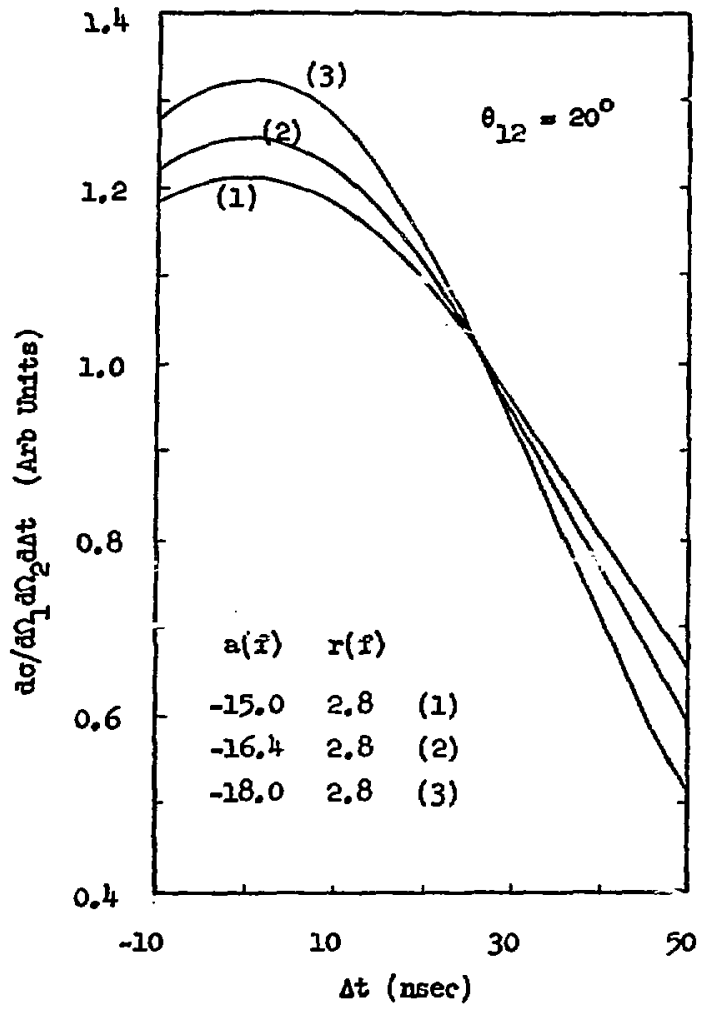

Fig. 3. Two-neutron differential time-of-filight spectra normalized to unit area over the symetric interval $-50 \leqslant \Delta t<+50$ plotted for $\theta_{12}=20^{\circ}$ and various values of $a_{n n}$. Flight path equal $3 m$.

optimal opening angle in the neighborhood of $10^{\circ}$ and an optimel range in $\Delta t$ of $\pm 100 \mathrm{nsec}$.

The more interesting question is: What ere the theoretical uncertainties in extracting $a_{n n}$ using the theoretical procedures outlined above? A detailed discussion of the various problems that arise can be found in Appendix B. However, the main uncertainty lies in the sensitivity of the reaction to the unknown short-range properties of the $n-n$ final-state wave function. This is also the source of increased uncertainty that arises in extracting the Coulomb corrected $p-p$ scattering length from the proton-proton scattering data. Thus, the most meaningful analysis in terms of looking for charge asymmetry in the $\mathrm{N}-\mathrm{N}$ force would involve a simultaneous analysis of the $p-p$ scattering data along with the $\mu$-capture data. In this way the same uncertainty in the short-range character of the

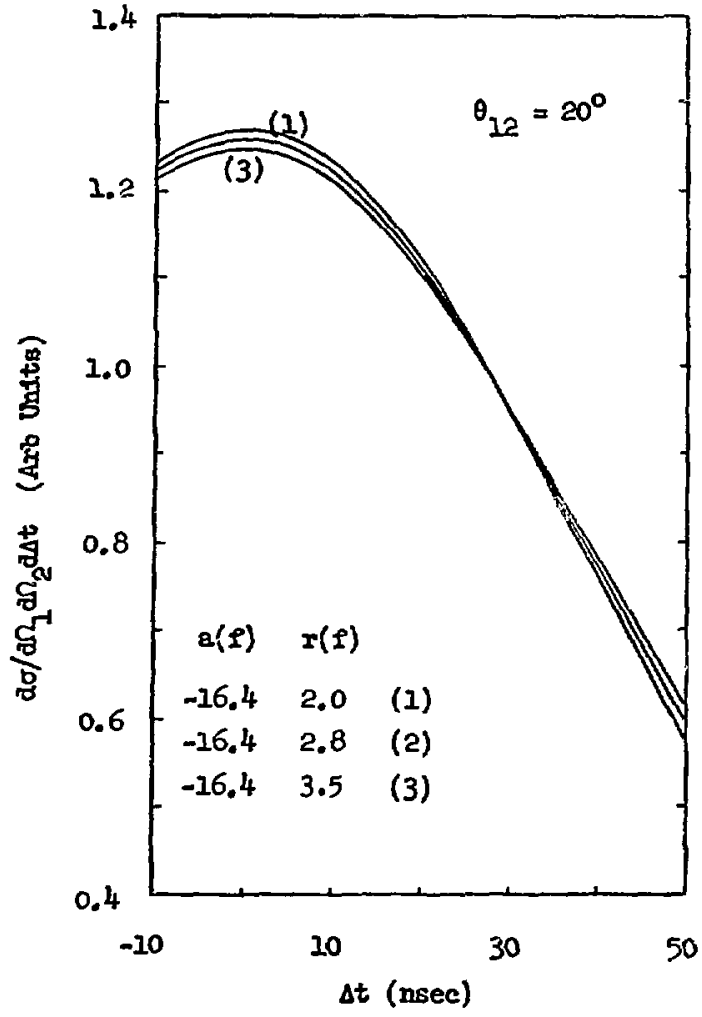

Fig. 4. Two-neutron differential time-of-flight spectra normalized to unit area over the symmetric interval $-50<\Delta t \leqslant+50$ plotted for $\theta_{12}=20^{\circ}$ and various values of $r_{n n}$. Flight path equal $3 \mathrm{~m}$.

scattering state would appear in each analysis.

\section{CONCLUSIONS}

Because the weak interaction is thought to be well understood, the $\mu$-capture reaction offers the cleanest theoretical interpretation of the three competing reactions:

$$
\begin{aligned}
& \mu^{-}+d+n+n+v \\
& \pi^{-}+d+n+n+\gamma \\
& n+d+n+n+p
\end{aligned}
$$

However, the largest theoretical uncertainty in the determination of $a_{n n}$ arises from the unknown short-range character of the $n-n$ final-state wave function. This uncertainty is common to all three reactions.

Since the $\mu$-capture exferiment entails a differential time-of-flight measurement and the count 
rate is much lower than for the analogous radiative $\pi$-capture experiment, it would seem that the difficulty assocsated with $\mu$-capture relative to the difficulty associated with radiative $\pi$-capture is large. Because the some source of theoretical un- certainty dominates our analysis of both of thes? experiments for determining $a_{n n}$, it would appear that the $\pi$-capture experiment is preferable. However, a comparison of the two reactions rould provide a zeasonable check on the theoretical assumption concerning the $\pi-d$ initial state.

APPENDIX A

The low-energy scattering parameters for the singlet neutron-proton and the proton-proton systems are known to high accuracy: ${ }^{13-15}$

${ }_{p p}^{c}=-7.823 \pm .01 \mathrm{f}, \quad r_{p p}^{c}=2.794 \pm .015 \mathrm{f}$, $a_{n p}^{s}=-23.715 \pm .015 \mathrm{f}, r_{n p}^{s}=2.73 \pm .13 \mathrm{f}$.

The superscript $C$ designates that these are the measured $p-p$ quantities which include Coulomb effects. The uncertainty associated with $a_{p p}{ }^{c}$ is certainly very small. However, it is the "Coulomb corrected" value of the $p-p$ scattering length that is needed for comparison with the $n-n$ value. The removel of Couromb effects is a model-dependent process. ${ }^{16}$ Thus the uncertainty in the estimate of $a_{p p}$ is much larger than the experimental uncertainty in ${ }_{\mathrm{ap}}^{\mathrm{c}}$. A generally accepted estimate for the Coulomb corrected scattering length and effective range is 17,18

$a_{p p}=-17.1 \pm .3 \mathrm{f}, \quad r_{p p}=2.84 \pm .03 \mathrm{f}$.

At present the $n-n$ scattering length is very uncertain. The most recentiy quoted average value (from the conference on few nucleon probisms held at UCLA) is 18,19

$$
a_{n n}=-16.3 \pm .9 \mathrm{f} .
$$

The uncertainty in $r_{n n}$ is much greater:

$$
r_{n n}=2.8 \pm .5 \mathrm{f} \text {. }
$$

The possibility that the $\mathbb{N}-N$ interaction is charge symmetric is certainly included within the quoted errors. However, it is quite elear that a precise determination of $a_{n n}$ is lacking.

\section{APPENDIX B}

In orier to acquire some understanding of the sensitivity of the $n-n$ scattering length to various uncertalnties in the theory of the $\mu^{-}+d-$ $n+n+v$ reaction, we have analyzed our optimum theoretical model of the $n-n$ differential time-offlight spectra by modifying the model to account for one or more uncertain aspects; e.g., alterins the effective range. The error in the extrected scattering length in such an analysis is then a measure of the theoretical uncertainty characterizing that aspect of the theory being investigated. No attempt has been made to analyze all possible theoretical uncertainties; rather it was attenpted to make $a$ thorough arialysis of the most important problems.
The model for the deuteron was taken to be the RSC wave function 17 including the D-state component. However, to facilitate the error analysis discussed below, only the renormalized S-state component of the wave function was used; the error in the scattering length due to such an approximation can be seen from Table B-I below to be smell. The uncertainty in $a_{n n}$ due to the choice of this model for the ground state was tested by analyzing with the Hulthen form for the deuteron wave function.

The uncertainty in $a_{n n}$ resulting from the unknown value of $F_{P}$ was determined by varying $F_{P}$ from its assumed value of $7 F_{A}$ to one of $8 F_{A}$. Such a variation in the coupling constant had only a negligible effect on the extracted scattering 
length. This resulted from our restriction of the kinematics to a smail region of relative n-n momentum.

The N-N final state was represented by a PRS model ${ }^{11}$ in which the phase shift was fixed and the wave function was obteined by integrating the Schrödinger equation in over a known local potential (e.g., the RSC singlet $s_{0}$ potential) to a radius of one pion Cormpton wavelength $(R)$, where the wave function and first two derivatives were matched to determine a smooth polynomial fit into the origin $\left(r \psi=(r \psi)^{\prime}=(r \psi)^{\prime \prime}=0\right.$ at $\left.r=0\right)$; a fifth-order polynomial gives a uniqu? fit to the six boundary conditions. To allow for uncerteinty in our knowledge of the short-range part of the wave function inside the matching radius, a function of the form $\eta(p) r^{3}(r-R)^{3}$ wes added to the polynomial; cleariy variation in the assumption made about the function $\eta(p)$ will modify the spectra and the value extracted for ${ }^{a}$. The PRS model value corresponds to $\eta(p)=0$; the model sensitivity was investigated by analyzing the spectre with $\Pi(p)$ determined by matching the third derivative at $R$ and by assuming $\eta(p)=\$ 5.0 \mathrm{p}$. In addition, the uncertainty in ${ }_{\mathrm{nn}}$ due to the unknown effective range $r_{n n}$ used to determine the s-wave phase shift

$$
p \cot \left(8_{0}\right)=\frac{-1}{a_{n n}}+\frac{1}{2} r_{n n} p^{2}
$$

was tested by modifying the effective from its assumed value of $2.8 \mathrm{f}$.

In the model calculations it was assumed that the only nonzero phase shift of consequence occurred in the $l=0$ partiel wave. (All higher multipole contributions were included in the calculetion assuming $\left.b_{\ell}=0, \ell \neq 0.\right)$ In order to investigate the uncertainty due to this assumption, a p-wave phase shift of $\delta_{1}=0.1 p^{2}$ was used to analyze the spectre. The results of the calculations showed that although the higher multipoles are important, the uncertainty in the phase shifts in these partial waves is not significant.

In Table B-I are shown the results of our examination of the theoretical uncertainty in the extraction of the $n-n$ scattering length. Note that as discussed in the text, the smaller opening angle provides a larger range in relative momentum $p$ to be investigated for a given time interval. At $30^{\circ}$ the

THE ERROR (IN f) IN a nn EXTRACTED FROM THE IIFFERENTIAL TIME-OF-FLIGHT SPECTRA DUE TO THE MODEL ERROR NOTED IN THE FIRST COLUMN AS A FUNCTION OF OPENING ANGLE FOR $\Delta t=50$ NSEC.

\begin{tabular}{|c|c|c|c|c|c|}
\hline & $\theta_{12}$ & $=$ & $10^{\circ}$ & $20^{\circ}$ & $30^{\circ}$ \\
\hline RSC with D--state & & +0.008 & +0.010 & +0.020 & +0.042 \\
\hline Hulthén bound state & & +0.001 & +0.001 & +0.003 & +0.007 \\
\hline$F_{P}=8 F_{A}$ & & - & - & - & - \\
\hline$\delta_{p}=0.1 p^{2}$ & & -0.008 & -0.007 & -0.006 & +0.001 \\
\hline $\mathrm{r}_{\mathrm{nn}}=2.6 \mathrm{f}$ & & +0.079 & +0.076 & +0.065 & +0.037 \\
\hline$n(p) \sim(r \psi)^{\prime \prime \prime}$ & & -0.006 & +0.002 & +0.003 & +0.007 \\
\hline$\eta(p)=5 p$ & & -0.031 & -0.045 & -0.063 & -0.098 \\
\hline$n(p)=-5 p$ & & +0.037 & +0.040 & +0.060 & +0.097 \\
\hline zero range scattering state & & -0.006 & -0.010 & -0.033 & -0.101 \\
\hline Hulthén + zero range scat. state & & -0.015 & -0.017 & -0.041 & -0.117 \\
\hline $\begin{array}{l}\text { Hulthén }+ \text { zero range scat. state } \\
(l=0 \text { multipole only })\end{array}$ & & -0.069 & -0.103 & -0.229 & -0.653 \\
\hline
\end{tabular}


range in $p$ shown here is so omall as to make the anelysis insensitive to $r_{n n}$; however, the sensitivity to $a_{\mathrm{m}}$ is also lost (1.en, the chi-square curve becomes yery flat making the uncertainty in $a_{n n}$ very large). Note also that omitting the higher multipoles entirely can produce a large error in the extracted scattering length. It should be pointed out that any momentum dependent correction to the theory (i.e., variation of the phase shift with $p$ due to an uncertainty in $r_{n n}$ as opposed to a constant change from an $F_{P}$ of $7 F_{A}$ to one of $\left.8 F_{A}\right)$ will produce a much larger effect on the

\section{REFERENCES}

1. H. Uberall and I. Wolfenstein, "Capture of $\mu-$ Mesons in Deuterium", Nuovo Cimento 10,136 (1958).

2. H. G. Dosch, "The Influence of the NeutronNeutron Final-State Interaction on Cafcure or Muons by Deuterons", Nuovo Cimento 39, 731 (1965).

3. I. T. Wang, "Muon Capture by Deuterons", Phys. Rev. 139 , B1539 (1965).

4. E. Cremer, "Relativistic Treatment of Muon Capture in Deuterium", Nucl. Phys. B2, 409 (1967); errors in this reference are pointed out in Ref. 6 .

5. E. Truhlik, "Neutron-Neutron Interaction and Muon Capture by Deuteron", Nucl. Fhys. 145 , $303(1972)$.

6. P. Pascual, R. Tarrach, and F. Vidal, "Muon Cepture in Deuterium", Nuovo Cimento 12A, 241 (1972).

7. I. T. Wane, E. W. Anderson, E. J. Bleser, L. Lederman, S. L. Mayer, J. L. Rosen, and J. E. Rothbers, "Muon Capture in (pud) ${ }^{+}$Molecules", Phys. Rev. 139, B1528 (1965).

8. A. Fujii and H. Primakoff, "Muon Capture in Certain Light Nuclei", Nuovo Cimento 12, 327 (1958).

9. H. Primakoff, "Theory of Muon Capture", Rev. Mod. Phys. 31, 802 (1959).

10. C. W. Kim and H. Primakoff, "Theory of Muon Capture with Initial and Final Nuclei Treated es "Elementary' Particles", Phys.Rev. 140, B566 (1965). scattering length than a correction that is not momentum dependent.

Fror the numerical results in Table $\mathrm{B}-\mathrm{I}$ one can see that the theoretical uncertainty in a careful analysis of the $\mu^{-}+a \rightarrow n+n+\nu$ reaction could be mede less than some $0.2 \mathrm{f}$. Since we have not explored all of the possible theoretical problems that might contribute to the uncertainty, a better estimate of its range might be estimated to be in the neighborhood of $0.3 \mathrm{f}$. However, it should be cleer thet the theoretical uncertainty in the extraction of $a_{\mathrm{nn}}$ is smaller than the present experimental error.

11. H. S. Picker, E. F. Redish, and G. J. Stephenson, Jr., "Two-Nucleon T Matrix Half off the Energy Shell: A Direct Approach", Phys. Rev. C $\underline{4}, 287$ (1971).

12. M. Leon and $\mathrm{H}$. Bethe, "Negative Meson Absorption in Liquid Hydrogen", Phys. Rev. 127, $636(1962)$.

13. H. P. Noyes, "The Nucleon-Nucleon Effective Range Expansion Parameters", Ann. Rev. Nucl. Sci. 22, 465 (1972).

14. H. $\mathrm{P}_{1}$ Noyes and $\mathrm{H} . \mathrm{M}$. Lipinski, "Comment on the ${ }^{1}{ }_{S}$ Nucleon-Nucleon Effective-Range Expansion Parameters", Phys. Rev. C 4, 995 (1972).

15. M. S. Sher, P. Signell, and L. Heller, "Characteristics of the Proton-Proton Interaction Deduced from the Data Below $30 \mathrm{MeV}$.", Ann. of Phys. 58, 1 (1970).

16. I. Heller, P. Signell, and N. R. Yoder, "Charge Symmetry, Charge Dependence, and the NucleonNucleon Scattering Lengths", Phys. Rev. Lett. 13, 577 (1964).

17. R. V. Reid, "Local Phenowenological NucleonNucleon Potentials", Ann. of Phys. 50, 411 (1968).

18. E. M. Henley and D. H. Wilkinson, Few Particle Problems in the Nuclear Interaction, Ed. by I. Slaus, S. A. Moszkowski, R. P. Haddock, and W. T. H. van Oers (North-Holland Publ. Co., Ansterdam, 1972) pp. 229-33, 242-3.

19. R. M. Salter, M. Zeller, R. P. Haddock, D. Nygren, and J. B. Cairr, Few Particle Problems in the Nuclear Interaction, Ed. by I. Sleus, S. A. Moszkowski, R. P. Haddock, and W. T. H. van Oers (North-Holland Publ. Co., Ansterdam, 1972) pp. 112-113. 